\title{
Cortical white matter: beyond the pale
}

\author{
Kathleen S. Rockland ${ }^{1 *}$ and Javier DeFelipe ${ }^{2,3}$ \\ 1 RIKEN-MIT Center for Neural Circuit Genetics, Picower Institute for Learning and Memory, Massachusetts Institute of Technology, Cambridge, MA, USA \\ 2 Instituto Cajal, Consejo Superior de Investigaciones Científicas, Madrid, Spain \\ ${ }^{3}$ Centro de Tecnología Biomédica, Universidad Politécnica de Madrid, Madrid, Spain \\ *Correspondence: kathrock@mit.edu
}

The tracts within the subcortical white matter and corpus callosum provide an anatomical connectivity that is essential for normal cognitive functioning. These structures are predominantly made up of axons that are myelinated or unmyelinated, and entering or exiting the overlying gray matter. As is increasingly recognized, however, the white matter territory is neither inert nor static. It has its own microenvironment, consisting of scattered neurons, abundant glia, and blood vessels; but at the same time it is an integrated component with the much more neuron dense gray matter.

This volume brings together 10 articles that are intended to provide a summary of some of the current thinking regarding white matter organization and the myelination process, including implications for normal and abnormal brain processes. A final article (DeFelipe et al.) is organized as a series of extended extracts and commentaries of the individual articles, each of which is followed by general comments and discussion of some of the issues raised. The order of articles in the present volume parallels the order in this final article. Topics are briefly described below.

The first two articles (Suarez-Sola et al. and Friedlander and Torres-Reveron) focus on the population of neurons in the white matter (see also Clancy et al., 2010). These are identified as "interstitial neurons," although also called by other identifiers. They are heterogeneous, and are proposed in the second of these articles to subserve varying functions, undergoing temporal re-specification of function over the lifespan. In Jakovcevski et al. the authors review the complex interplay of oligodendrocyte development, the expression of myelin proteins, and the formation and restoration of myelin. Important species differences between human and rodents are discussed. A fourth article, by Jovanov-Milosevic et al., focuses on the developmental progression of axons within the human corpus callosum, at both the molecular and morphological levels.

Another cluster of articles addresses structural-functional relationships between white matter tract microstructure and cognitive abilities. Niogi et al. provide evidence for three separable attentional networks, correlated with anatomically distinguishable networks. Paus and Toro discuss anatomical changes in the white matter, particularly as these are associated with the adolescent period, and propose a model where the $g$ ratio (related to the sum of the axon diameter and thickness of the myelin sheath) may be selectively impacted in large fibers, under the influence of testosterone, with pathological consequences. Kimura and Itami describe variability in axon diameter and degree of myelination, relating this to areaspecific differences in conduction velocity and implications for network integration.

Finally, white matter has long been well-known to figure prominently in multiple disease states. The article by Peters brings together data on the ultrastructural changes during normal aging of myelinated axons in the macaque, treating both underlying mechanisms and functional consequences in the context of cognitive impairment. In Hoistad et al. the authors discuss several lines of research in support of the premise that dysfunction of oligodendrocytes, triggering a cascade of myelin-related malfunctions, is a critical factor in the development of schizophrenia. The article by Lee and Fields emphasizes the active intercommunication of axons and glia, the complex interplay of gene expression and neural activity, and the implications for psychiatric disorders.

In summary, these articles present a range of data and issues that are under active investigation in the field and closely related to basic issues of white matter organization in normal and pathological conditions. We hope the reader will find this collection a useful guide to both navigating the extensive background in this area of research and interpreting the exciting new results that can be anticipated.

\section{REFERENCE}

Clancy, B., DeFelipe, J., Espinosa, A., Fairen, A., Jinno, S., Kanold, P., Luhmann, H. J., Rockland, K. S., Tamamaki, N., and Yan, X. (2010). Cortical GABAergic neurons: stretching it remarks, main conclusions and discussion. Front. Neuroanat. 4:7. doi: 10.3389/neuro.05.007.2010

Received: 13 December 2011; accepted: 16 December 2011; published online: 10 January 2012.

Citation: Rockland KS and DeFelipe J (2012) Cortical white matter: beyond the pale. Front. Neuroanat. 5:67. doi: 10.3389/fnana.2011.00067

Copyright (C) 2012 Rockland and DeFelipe. This is an open-access article distributed under the terms of the Creative Commons Attribution Non Commercial License, which permits non-commercial use, distribution, and reproduction in other forums, provided the original authors and source are credited. 